Bundesgesundheitsbl $2021 \cdot 64: 625-634$ https://doi.org/10.1007/s00103-021-03312-1

Eingegangen: 18. November 2020

Angenommen: 12. März 2021

Online publiziert: 14. April 2021

(c) Der/die Autor(en) 2021

\author{
Robin Rüsenberg' $\cdot$ Axel Baumgarten ${ }^{1,2} \cdot$ Stefan Mauss ${ }^{1,3} \cdot$ Gabriele Gradl $^{4}$. \\ Martin Schulz ${ }^{4,5,6}$ - Barbara Bartmeyer ${ }^{7}$. Christian Kollan ${ }^{7}$. Daniel Schmidt ${ }^{7}$ \\ ' Deutsche Arbeitsgemeinschaft niedergelassener Ärzte in der Versorgung HIV-Infizierter e. V. (dagnä), \\ Berlin, Deutschland \\ ${ }^{2}$ Zentrum für Infektiologie Berlin Prenzlauer Berg (ZIBP), Berlin, Deutschland \\ ${ }^{3}$ Zentrum für HIV und Hepatogastroenterologie, Düsseldorf, Deutschland \\ ${ }^{4}$ Deutsches Arzneiprüfungsinstitut e. V. (DAPI), Berlin, Deutschland \\ ${ }^{5}$ Institut für Pharmazie, Freie Universität Berlin, Berlin, Deutschland \\ ${ }^{6}$ Geschäftsbereich Arzneimittel, ABDA - Bundesvereinigung Deutscher Apothekerverbände e. V., Berlin, \\ Deutschland \\ ${ }^{7}$ Abteilung für Infektionsepidemiologie, Robert Koch-Institut, Berlin, Deutschland
}

\title{
Wie wirken Generikaquoten? Eine Analyse am Beispiel der HIV- Infektion
}

neuordnungsgesetz)-Verfahren, das durch eine frühe Nutzenbewertung und anschließende Erstattungsbetragsverhandlungen gekennzeichnet ist. Von 2011 bis Ende 2020 gab es 21 Nutzenbewertungen im Bereich HIV, deren inhaltliche Ergebnisse auch direkt das Verordnungsverhalten der Schwerpunktärzte beeinflussen [1]. Ebenfalls den Preis nehmen die bereits seit 1989 existierenden Festbeträge in den Fokus. Im Jahr 2016 hat der Gemeinsame Bundesauschuss (G-BA) mit der Bildung von Festbeträgen für die HIV-Indikation begonnen (Zidovudin + Lamivudin, Efavirenz) - noch ohne größere Auswirkungen auf die Versorgungsrealität. Abzuwarten bleibt, wie die neue Festbetragsgruppe „Kombinationen zweier Nukleos(t)id-Analoga“ (Tenofovir-Disoproxil/Emtricitabin TDF/FTC, Abacavir/Lamivudin $\mathrm{ABC} / 3 \mathrm{TC}$ und TenofovirAlafenamid/Emtricitabin TAF/FTC) sowie des Proteaseinhibitors Darunavir (DRV) seit Oktober 2020 wirkt [2].

Bereits umfangreichere Erfahrungen gibt es in der HIV-Schwerpunktversorgung mit (ebenfalls preisregulierenden) Rabattverträgen, die in der Regel bei der Abgabe in der Apotheke ansetzen. Mittlerweile informieren zudem Krankenkassen verstärkt die HIV-Schwerpunktärzte über die von ihnen abge- schlossenen Rabattverträge. Zusätzlich existieren vielfältige Instrumente der regionalen Verordnungssteuerung, die den Fokus auf Struktur und/oder Menge und damit vor allem auf Verhaltenssteuerung bei dem verordnenden Arzt setzen. $\mathrm{Zu}$ erwähnen sind vor allem die seit 2020 über die jeweilige regionale Kassenärztliche Vereinigung (KV) eingeführten Generikaquoten für HIV-Therapeutika in Bayern und Berlin. Auf der Ebene der regionalen Arzneimittelvereinbarungen zeigt sich damit immer deutlicher ein $\mathrm{Pa}$ radigmenwechsel: einerseits eine Abkehr von statistischen Auffälligkeitsprüfungen, etwa Richtgrößen oder anhand von Durchschnittswerten, andererseits eine Hinwendung $\mathrm{zu}$ indikationsbezogenen Quoten, vorrangig Leitsubstanzen und/ oder Generika/Biosimilars.

Eine entscheidende Rolle bei der Arzneimittelregulierung durch Quoten nimmt, wenig überraschend, das Verordnungsverhalten der Vertragsärzte ein. Ziel des vorliegenden Beitrages ist es, auf Grundlage von GKV-Apothekenabrechnungsdaten das Potenzial sowie Grenzen von Generikaquotenlösungen in der Versorgung von Menschen mit HIV zu analysieren und Handlungsempfehlungen zu skizzieren. Dabei sollen ebenfalls der (nachfragesteuernde) Status quo in der HIV-Arzneimittelversorgung so- 
wie das Instrument der Rabattverträge beschrieben werden.

\section{HIV-Arzneimittelversorgung und verordnungssteuernde Maßnahmen}

Die ambulante HIV-Schwerpunktversorgung in Deutschland ist überaus erfolgreich - der medizinische Goldstandard, also die effektive Absenkung der Viruslast im Blut unter die Nachweisbarkeitsgrenze, wird bei $96 \%$ der Betroffenen in Therapie erreicht [3]. Die Versorgung ist aber auch kostenintensiv. Im Schnitt hatte die GKV im Jahr 2018 Leistungsausgaben von ca. $3100 € / \mathrm{Jahr}$ pro Versicherten [4]; die Versorgung von Patienten, die mit HIV infiziert sind, ist finanziell aufwendiger. Für HIV-Patienten sind es - bezogen auf den Zeitraum 2014-2017 - im Schnitt 17.300€/Jahr, woran die HIV-Therapeutika einen Anteil von ca. $85 \%$ haben [5]. Seit 2018 sind die Kosten infolge von Preisanpassungen gesunken.

Die ART-Standardtherapie setzt als Kombinationstherapie i.d.R. auf 3 antiretrovirale Substanzen aus mindestens 2 Wirkstoffklassen der $\mathrm{Nu}$ kleos(t)ide-Reverse-Transkriptase-Inhibitoren (NRTI) sowie entweder NonNukleos(t)ide-Reverse-TranskriptaseInhibitoren (NNRTI), Proteaseinhibitoren (PI) oder Integrase-Strang-TransferInhibitoren (INSTI; [6]). Mittlerweile werden diese oftmals zusammen in einer Tablette verabreicht (Eintablettenregime, Single-Tablet-Regimen - STR). Dies zeigt sich auch in gesunkenen Absatzmengen bezogen auf die Tagesdosen [7]. Seit Kurzem wurden zusätzlich 2 duale STR-Regime im Markt platziert, die mittlerweile einen nennenswerten Umsatz haben. Anfang 2020 wurden 40 Präparate zur Behandlung der HIVInfektion in Deutschland verordnet, von denen wiederum knapp ein Drittel generisch verfügbar war. Es gilt allerdings zu bedenken, dass der Einsatz der meisten ART-Generika - die es grundsätzlich seit 2013 gibt - nicht mehr durch die Leitlinien empfohlen wird [8]. Vor allem seit dem Fall des Patents von TDF bzw. TDF/FTC im Juli 2017 ist eine deutliche Marktdynamik zu spüren, die sich in gesunkenen Tagestherapiekosten niederschlägt. TDF ist Bestandteil von Erstlinientherapeutika und der Präexpositionsprophylaxe der HIV-Infektion (PrEP). Anfang 2019 lag die ART-Generikaquote bei knapp über $20 \%$ [1, 9, 10].

Unmittelbar auf das generische Segment zielen Rabattverträge nach $\$ 130 \mathrm{a}$ Abs. 8 Fünftes Buch Sozialgesetzbuch (SGB V). Diese erfreuen sich bei Krankenkassen großer Beliebtheit, da sie direkte Einsparungen genieren (2019: 4,9 Mrd. €; [11]); sie werden auch in der privaten Krankenversicherung (PKV) genutzt. Als angebotsorientiertes Steuerungsinstrument setzen sie beim Faktor Preis an und werden als Teil des Vertragswettbewerbs direkt zwischen Krankenkasse und pharmazeutischem Unternehmen vereinbart. Den entscheidenden Schub erhielt das Instrument Rabattverträge durch die seit 2007 geltende Verpflichtung der Apotheken, die kassenspezifischen Rabattverträge vorrangig zu bedienen, sofern der Verordnende den Austausch (durch Verzicht auf das Setzen eines Aut-idem-Kreuzes in der Verordnung) zugelassen hat [12]. Auch im Segment der HIV-Therapeutika existiert eine Vielzahl an Rabattverträgen. Detaillierte Aussagen sind aufgrund der Vertraulichkeit der Vereinbarungen allerdings schwer zu treffen. Über das Arztinformationssystem wird der Verordnende aber über das Vorhandensein eines Rabattvertrages informiert. Auch die einzelnen Krankenkassen informieren darüber. Das Vorhandensein von Rabattverträgen hat schließlich bei fast $66 \%$ der HIV-Schwerpunktpraxen Einfluss auf das Verordnungsverhalten [1]. Hilfreich ist, dass Krankenkassen für rabattierte Arzneimittel i.d.R. eine „Wirtschaftlichkeitsgarantie“ geben, d.h., die Verordnung gilt immer als wirtschaftlich, wodurch der verordnende Arzt vor Regressforderungen durch die Krankenkasse geschützt ist [13].

Auch in den regionalen Arzneimittelvereinbarungen nach $\$ 84$ SGB V finden Rabattverträge oft Berücksichtigung. Diese sind das zentrale Instrument der Nachfragesteuerung. Zwar existieren ebenso Informationsschreiben von KV und/oder Krankenkassen, im Mit- telpunkt stehen jedoch die regional zwischen KV und den Landesverbänden der Krankenkassen und den Ersatzkassen vertraglich festgelegten Regelungen. Diese werden durch regelmäßige Wirtschaftlichkeitsprüfungen nach $\$ 106$ SGB V umgesetzt. Bis Ende 2016 war die sog. Richtgrößenprüfung (Auffälligkeitsprüfung) als Regelprüfmethode vorgesehen, also ein arztgruppenspezifisches, statistisches Prüfverfahren mit normativem Charakter. Die HIV-Therapie war in allen KV-Regionen dabei als Praxisbesonderheit definiert, um dem Umstand Rechnung zu tragen, dass die ART in den Schwerpunktpraxen deutlich höhere Kosten verursachte als in den Praxen derselben Fachgruppe ohne HIV-Schwerpunkt (Stichwort „atypische Hausarztpraxen“). Ergänzend waren Höchst- und Mindestquoten, mal als Orientierung, mal mit Auswirkung auf die Richtgrößenprüfung, möglich. Etwa 2016 in der KV-Region Nordrhein, wo man sich den „Abbau von Fehl-, Überund Unterversorgung insbesondere im Bereich ... der Arzneimittel zur Therapie von HIV-Infektionen“ vornahm; allerdings ohne konkrete Umsetzungsschritte [14].

Zu Januar 2017 wurden die Rahmenbedingungen durch das GKV-Versorgungsstärkungsgesetz (GKV-VSG) regionalisiert. Seitdem ist die Richtgrößenprüfung nicht mehr obligatorisch vielmehr können die regionalen Vertragspartner eigene Prüfungsarten und -methoden umsetzen, was im Ergebnis zu einem bunten Strauß an verordnungssteuernden Maßnahmen geführt hat. Insgesamt zeigt sich jedoch ein Trend $\mathrm{zu}$ indikationsbezogenen Quoten, vorrangig Leitsubstanzen und/oder Generika/ Biosimilars (Zielquoten werden dabei selbst in denjenigen KV-Regionen, die noch auf Auffälligkeitsprüfungen setzen, als Ergänzung eingesetzt). Auch in der Versorgung von Menschen mit HIV findet dies langsam Niederschlag: Ende 2020 war in 15 von $17 \mathrm{KV}$-Regionen entweder - dort, wo nach statistischen Verfahren geprüft wird - HIV als Praxisbesonderheit anerkannt. Oder aber die Regelungen in den "Quoten“-Regionen sahen keine Vorgaben für die ART vor. 
Bundesgesundheitsbl 2021 • 64:625-634 https://doi.org/10.1007/s00103-021-03312-1

(c) Der/die Autor(en) 2021

R. Rüsenberg · A. Baumgarten · S. Mauss · G. Gradl · M. Schulz · B. Bartmeyer · C. Kollan · D. Schmidt Wie wirken Generikaquoten? Eine Analyse am Beispiel der HIV-Infektion

\section{Zusammenfassung}

Hintergrund. Die Steuerungsinstrumente der Arzneimittelversorgung in der gesetzlichen Krankenversicherung (GKV) sind schon länger Bestandteil der gesundheitspolitischen Reformdebatte. Über die Jahre hat sich eine Gemengelage sehr verschiedener Werkzeuge herausgebildet, die zumeist auf eine Kontrolle der Arzneimittelausgaben zielen. Die Instrumente der regionalen Verordnungssteuerung fokussieren vor allem auf eine Verhaltenssteuerung des verordnenden Arztes. Zu erwähnen ist nicht zuletzt der verstärkte Einsatz von indikationsbezogenen Quoten, vorrangig Leitsubstanzen und/oder Generika/Biosimilars. Diese gibt es mittlerweile auch im Bereich des Humanen Immundefizienz-Virus (HIV), etwa die seit
2020 in Bayern und Berlin eingeführten Generikaquoten für HIV-Therapeutika. Zielstellung. Ziel des vorliegenden Beitrages ist es, auf Grundlage von GKV-Apothekenabrechnungsdaten das Potenzial sowie Grenzen von Generikaquotenlösungen in der HIV-Versorgung zu analysieren und Handlungsempfehlungen zu skizzieren. Ergebnisse. Es zeigte sich, dass das Quotenpotenzial für Generika im patentfreien Bereich in der HIV-Versorgung bereits weitgehend ausgeschöpft wird. Dieser Umstand ist vor allem darauf zurückzuführen, dass die HIVVerordner den Austausch durch Verzicht auf Aut-idem-Kreuze auf dem Kassenrezept unterstützen.

Diskussion. Das steuerungspolitische Optimum ist fast erreicht. Dies ist auf das geeignete Instrumentarium zurückzuführen, das aus dem Rahmenvertrag zur Arzneimittelversorgung und einer leitliniengerechten Wirkstoffverordnung durch den Arzt besteht - in Verbindung mit dem AMNOG(Arzneimittelmarktneuordnungsgesetz)Verfahren und Festbeträgen. Leitlinienkonformität und (existierende) Eintablettenregime müssen beibehalten werden, damit die gute Versorgungsqualität gewährleistet bleibt.

\section{Schlüsselwörter}

Arzneimittelversorgung · Versorgungssteuerung · Ambulante Versorgung · Sekundärdaten · Gesetzliche Krankenversicherung (GKV)

\section{How do generic quotas work? An analysis using HIV infection as an example}

\section{Abstract}

Background. Instruments controlling statutory healthcare medical supply have long been a topic of debate in health policy reform discussions. Over the years, a variety of tools have been developed, most of which are aimed at controlling drug expenditure. The instruments controlling regional prescriptions primarily focus on controlling behavioural patterns of the prescribing physicians. Important to note is the increased use of indication-directed quotas, primarily of drug leads and/or generics/biosimilars. These are now also available in the area of the human immunodeficiency virus (HIV), such as the generic quotas for HIV medications introduced in Bavaria and Berlin in 2020.

Objective. The aim of this article is to analyse the benefits and limitations of generic quota solutions in HIV care using statutory health insurance drug prescription data and to outline recommendations for action. Results. It was observed that the quota potential for generics in the area of patentfree drugs in HIV care has already been largely exhausted. This can be explained by HIV prescribers supporting product exchange on the prescription.

Discussion. The best-case scenario in terms of regulation has almost been reached. This is due to a suitable set of instruments, including the framework agreement for medical supply as well as prescribing according to guidelines - in conjunction with the Pharmaceuticals Market Reorganisation Act (AMNOG) and reference prices for drugs. Conforming with guidelines and (existing) single-tablet regimens play an integral role in maintaining good quality of care.

\section{Keywords}

Medical supply - Managed primary care . Ambulatory healthcare $\cdot$ Secondary data . Statutory health insurance
Eine Ausnahme bildeten Bayern und Berlin: Die bayerische Wirkstoffvereinbarung sieht eine facharztgruppenspezifische Verordnungsquote für „HIV-Therapeutika“ vor (Generikaquote am ARTGesamtmarkt inklusive TDF zur Therapie der chronischen Hepatitis B [HBV] und TDF/FTC als PrEP). Jede Vergleichsgruppe erhält pro Wirkstoffgruppe eine Zielquote, z. B. $37 \%$ bei Hausärzten, $49,9 \%$ bei Internisten ohne Schwerpunkt, $89,2 \%$ bei fachärztlichen Internisten mit Spezialisierung Gastroenterologie sowie etwa $43 \%$ bei den meisten weiteren Arzt- gruppen, wobei Rabattverträge berücksichtigt werden [15]. Insgesamt werden arztgruppenspezifische Zielquoten definiert, deren Erfüllung weitere arztindividuelle Prüfungen obsolet macht.

In Berlin hingegen setzen die regionalen Vertragspartner zwar weiterhin auf ein statistisches Verfahren (Durchschnittswerteprüfung), allerdings fallen die meisten Praxisbesonderheiten inkl. HIV weg. Dafür wird für HIVSchwerpunktärzte eine Generikaquote von $17,86 \%$ am ART-Gesamtmarkt definiert [16], deren arztindividuelles
Erreichen dazu führt, dass die HIVTherapeutika komplett aus der Durchschnittswerteprüfung herausgenommen werden - und damit quasi wieder als Praxisbesonderheit gelten. Rabattverträge werden bei der Quotenerfüllung in Berlin nicht, dafür aber in Bayern berücksichtigt.

Bayern (reines Quotenmodell) und Berlin (Auffälligkeitsprüfung, aber Berücksichtigung von Quoten) stehen damit auch stellvertretend für die beiden Trends in der regionalen Verordnungssteuerung. Ein dritter Weg sind Selektiv- 
Tab. 1 Anteile Generika auf Basis der Defined Daily Doses (DDD) für HIV, Hepatitis B (HBV) und gesamt in Berlin, Bayern und den übrigen Regionen der Kassenärztliche Vereinigungen (KV) über die Jahre 2013-2020. (Datenquelle: Insight Health)

\begin{tabular}{|c|c|c|c|c|c|c|}
\hline & $\begin{array}{l}\text { HIV } \\
\text { DDD in Mio. }\end{array}$ & $\begin{array}{l}\text { HIV } \\
\text { Generisch (in \%) }\end{array}$ & $\begin{array}{l}\text { HBV } \\
\text { DDD in Mio }\end{array}$ & HBV & $\begin{array}{l}\text { Gesamt } \\
\text { DDD in Mio. }\end{array}$ & $\begin{array}{l}\text { Gesamt } \\
\text { Generisch (in \%) }\end{array}$ \\
\hline \multicolumn{7}{|c|}{ KV Bayern } \\
\hline 2013 & 4,60 & 0,5 & 0,85 & 5,2 & 5,46 & 1,2 \\
\hline 2014 & 4,73 & 1,5 & 0,91 & 4,8 & 5,65 & 2,0 \\
\hline 2015 & 4,61 & 2,3 & 0,93 & 5,1 & 5,54 & 2,8 \\
\hline 2016 & 4,57 & 2,6 & 1,01 & 5,5 & 5,58 & 3,2 \\
\hline 2017 & 4,58 & 4,6 & 1,09 & 28,4 & 5,67 & 9,2 \\
\hline 2018 & 4,31 & 12,0 & 1,14 & 59,2 & 5,45 & 21,9 \\
\hline 2019 & 4,5 & 25,4 & 1,24 & 81,4 & 5,74 & 37,4 \\
\hline 2020 & 2,31 & 30,9 & 0,63 & 85,4 & 2,94 & 42,5 \\
\hline \multicolumn{7}{|c|}{ KV Berlin } \\
\hline 2013 & 7,64 & 0,5 & 0,63 & 4,5 & 8,27 & 0,8 \\
\hline 2014 & 7,82 & 2,0 & 0,67 & 7,3 & 8,49 & 2,4 \\
\hline 2015 & 7,36 & 2,4 & 0,66 & 7,6 & 8,02 & 2,8 \\
\hline 2016 & 6,54 & 2,8 & 0,65 & 8,7 & 7,19 & 3,3 \\
\hline 2017 & 6,53 & 5,7 & 0,66 & 30,3 & 7,19 & 8,0 \\
\hline 2018 & 6,22 & 14,0 & 0,68 & 64,4 & 6,89 & 18,9 \\
\hline 2019 & 5,47 & 28,0 & 0,70 & 86,2 & 6,17 & 34,7 \\
\hline 2020 & 3,07 & 37,2 & 0,36 & 89,3 & 3,44 & 42,7 \\
\hline \multicolumn{7}{|c|}{ KV andere } \\
\hline 2013 & 22,63 & 0,7 & 5,06 & 3,1 & 27,69 & 1,1 \\
\hline 2014 & 23,04 & 2,5 & 5,48 & 6,3 & 28,52 & 3,2 \\
\hline 2015 & 23,05 & 3,2 & 5,69 & 8,6 & 28,74 & 4,2 \\
\hline 2016 & 23,96 & 3,7 & 6,06 & 9,5 & 30,01 & 4,9 \\
\hline 2017 & 23,84 & 6,4 & 6,38 & 29,0 & 30,22 & 11,1 \\
\hline 2018 & 22,22 & 14,8 & 6,64 & 62,7 & 28,86 & 25,8 \\
\hline 2019 & 20,7 & 25,4 & 7,00 & 83,0 & 27,7 & 39,9 \\
\hline 2020 & 11,63 & 30,9 & 3,63 & 87,9 & 15,27 & 44,5 \\
\hline \multicolumn{7}{|c|}{ Bundesweit } \\
\hline 2013 & 34,87 & 0,6 & 6,55 & 3,5 & 41,42 & 1,1 \\
\hline 2014 & 35,59 & 2,3 & 7,07 & 6,2 & 42,66 & 2,9 \\
\hline 2015 & 35,03 & 2,9 & 7,28 & 8,0 & 42,31 & 3,8 \\
\hline 2016 & 35,07 & 3,4 & 7,72 & 8,9 & 42,79 & 4,4 \\
\hline 2017 & 34,95 & 6,0 & 8,13 & 29,0 & 43,08 & 10,4 \\
\hline 2018 & 32,75 & 14,2 & 8,46 & 62,3 & 41,20 & 24,1 \\
\hline 2019 & 30,68 & 25,8 & 8,94 & 83,0 & 39,62 & 38,7 \\
\hline 2020 & 17,02 & 32,0 & 4,62 & 87,6 & 21,65 & 43,9 \\
\hline
\end{tabular}

verträge, um auf das Verordnungsverhalten des Arztes Einfluss zu nehmen. So ist im Oktober 2020 ein Selektivvertrag nach $\$ 140$ a SGB V zur Optimierung der Versorgung von Menschen mit HIV in der KV-Region Nordrhein in Kraft getreten, der u. a. einen Arzneimittelcheck vorsieht [17].

\section{Methodik}

Die im vorliegenden Beitrag genutzten Sekundärdatenquellen sind Verordnungsdaten aus Apothekenabrechnungszentren von Personen mit gesetzlicher Krankenversicherung. Dabei handelte es sich einmal um Daten des Dienstleisters INSIGHT Health GmbH \& Co. KG mit einer vom Anbieter angegebenen Abdeckung der öffentlichen Apotheken in allen Regionen Deutschlands von
$>99 \%$ sowie um Daten des Deutschen Arzneiprüfungsinstituts e. V. (DAPI) mit einer Abdeckung von mehr als $80 \%$ (bis Juni 2019) bzw. über $95 \%$ (ab Juli 2019), welche auf $100 \%$ hochgerechnet wurden. Die GKV repräsentiert rund $87 \%$ der deutschen Bevölkerung. Die ausgewerteten Daten enthielten abgerechnete Verordnungen der in Apotheken eingelösten Rezepte gegen die Infektionskrankheiten HIV und HBV. Bei der Behandlungsindikation ist $\mathrm{zu}$ 


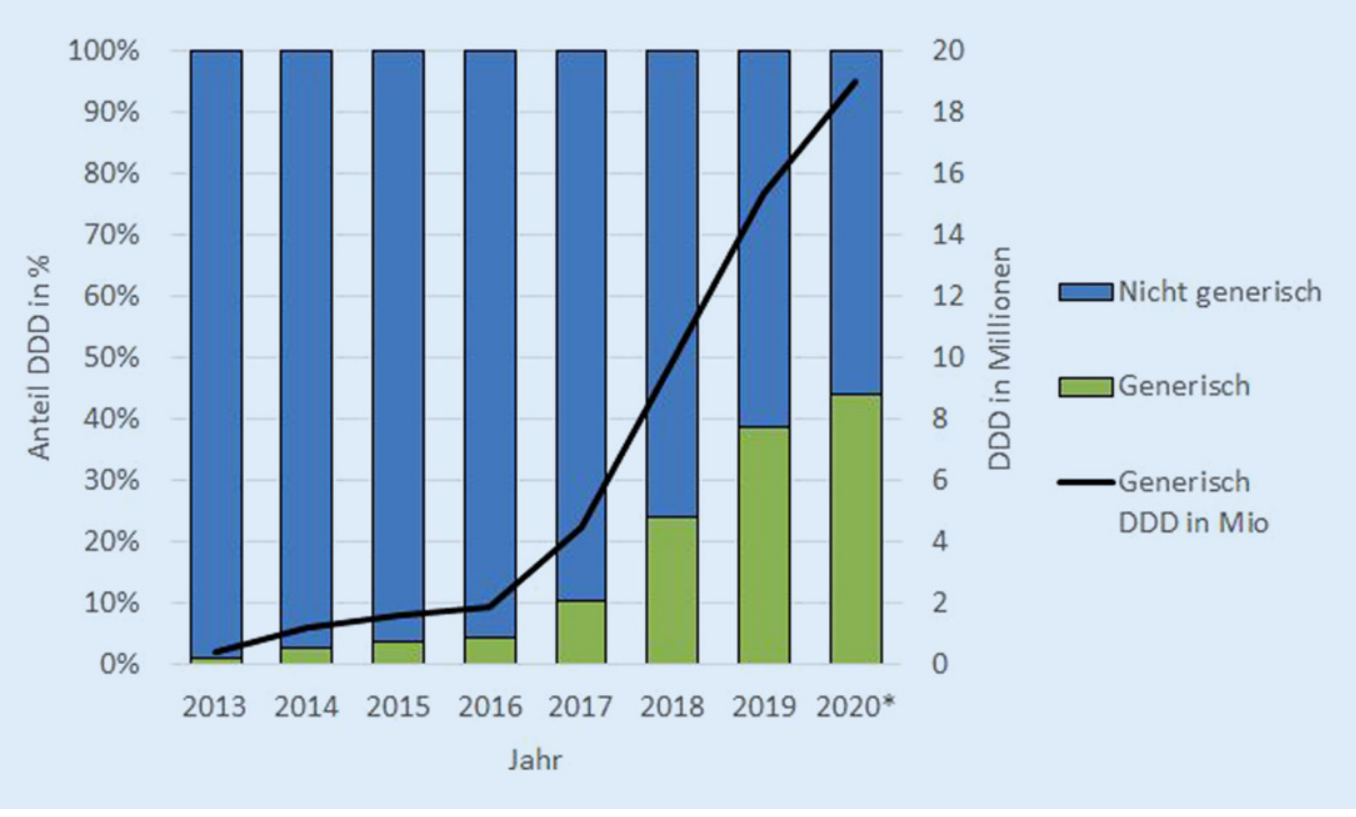

Abb. $1 \triangleleft$ Anteile generischer und nichtgenerischer Medikamente bundesweit über die Jahre 2013-2019. Summe Defined Daily Dose (DDD) für HIV, inkl. HIVPräexpositionsprophylaxe (PrEP) und Hepatitis B (HBV). ${ }^{*}$ Daten bis zum 1. Halbjahr 2020, die absoluten DDD für das 2. Halbjahr wurden auf Grundlage des 1. Halbjahrs geschätzt. (Datenquelle: Insight Health)

beachten, dass die Medikamente - TDF und die Kombination TDF/FTC - für 2 Einsatzgebiete die Marktzulassung besitzen: Ersteres für die Behandlung von HIV und HBV, Letzteres zur HIV-Behandlung als auch zur PrEP. Für welche Indikation verordnet wurde, lässt sich anhand der Abrechnungsdaten nicht ermitteln. In den weiteren Betrachtungen wird von der vereinfachenden, aber nicht ganz korrekten Annahme ausgegangen, dass TDF als Einzelsubstanz vollständig der HBV-Behandlung zuzuordnen ist.

Die Apothekenabrechnungsdaten wurden ausgewertet im Hinblick auf die Anteile von patentgeschützten Präparaten, Altoriginalen (nicht mehr patentgeschützte Erstanbieterprodukte), hierzu verfügbaren Generika. Zudem wurden sie auf bestimmte Steuerungsinstrumente wie den Rahmenvertrag über die Arzneimittelversorgung nach $\$ 129$ Abs. 2 SGB V (Abgaben von Rabattarzneimitteln, Abgaben mit gesetztem Autidem-Kreuz) untersucht. Ziel ist es, auf Basis der Fragestellungen des Beitrages das Verordnungsverhalten der jüngeren Zeit abzubilden. Es wurde zunächst ein Blick auf den Status quo für den Zeitraum 2013 bis 2020 vorgenommen. Die Anteile von Generika am Gesamtmarkt auf Basis der definierten Tagesdosen (Defined Daily Dose [DDD]) für HIV und $\mathrm{HBV}$ wurden in Berlin, Bayern und den übrigen KV-Regionen ermittelt. ${ }^{1}$ Darüber hinaus wurden Quotenszenarien unter Berücksichtigung der aktuellen Deutsch-Österreichischen Leitlinien zur antiretroviralen Therapie der HIV-Infektion [8] entwickelt, um den Einfluss einer Generikaquote auf die Therapiewahl zu prüfen.

\section{Ergebnisse}

In - Tab. 1 ist der generische Anteil an den jährlichen Gesamt-DDDs für die KV Bayern, Berlin und die weiteren $15 \mathrm{KV}$ Regionen nach Krankheitsindikation dargestellt. Hierbei ist $\mathrm{zu}$ bedenken, dass in der bayerischen Wirkstoffvereinbarung zwar von HIV-Therapeutika gesprochen wird, aber für die Generikaziele in dieser Kategorie alle $\mathrm{Me}$ dikamente sowohl für die Behandlung und Prophylaxe von HIV als auch der HBV zusammengefasst wurden (Spalte „Gesamt“).

Es war ein deutlicher Anstieg der Generikaquote über die letzten Jahre zu verzeichnen (• Abb. 1). Im Jahr 2020 lag die Generikaquote im HIV-Bereich in Bayern und dem restlichen Bundesgebiet bei $31 \%$, in Berlin bei $37 \%$. Trotz

\footnotetext{
1 Das Manuskript wurde im November 2020 eingereicht und konnte die in 2021 für die KV-Region Hamburg eingeführte Generikaquote daher nicht berücksichtigen.
}

des sprunghaften Anstiegs von generischem TDF/FTC sowohl in der HIVTherapie als auch durch die PrEP seit September 2019 liegt die erreichte Quote für den HIV-Bereich im Jahr 2020 bei unter $40 \%$. Aufgrund des starken Anteils des seit 2017 generischen TDF in der HBV-Therapie und der Tatsache, dass auch Entecavir generisch ist, wurden bei dieser Indikation nun Quoten von bis $\mathrm{zu}$ fast $90 \%$ erreicht. Damit wurde für die Gesamtheit der HIV- und HBVTherapeutika eine Quote von $44 \%$ im Jahr 2020 erreicht (• Tab. 1; • Abb. 1). Bei einer vollständig generischen Gabe der $\mathrm{HBV}$-Therapie steigt die generische Gesamtquote über alle 3 Indikationen noch um $3 \%$.

Welche aktiven Handlungsoptionen bieten sich nun aber unter den Vorgaben einer Arzneimittelvereinbarung, deren zentrale Steuerungselemente Generikaquoten und Rabattverträge sind? Wie erwähnt gilt für Apotheken die Verpflichtung, die kassenspezifischen Rabattverträge vorrangig $\mathrm{zu}$ bedienen, sofern der Verordnende den Austausch zulässt. Es wurden also die Fälle betrachtet, in denen dem Apotheker vom Verordnenden explizit untersagt wurde, Medikamente gegen ein wirkstoffgleiches Präparat zu tauschen ( $\bullet$ Abb. 2). Das im gegenteiligen Wortsinn sogenannte Aut-idem-Kreuz auf dem Rezept kann sich, je nach Wahl, sowohl im positiven 


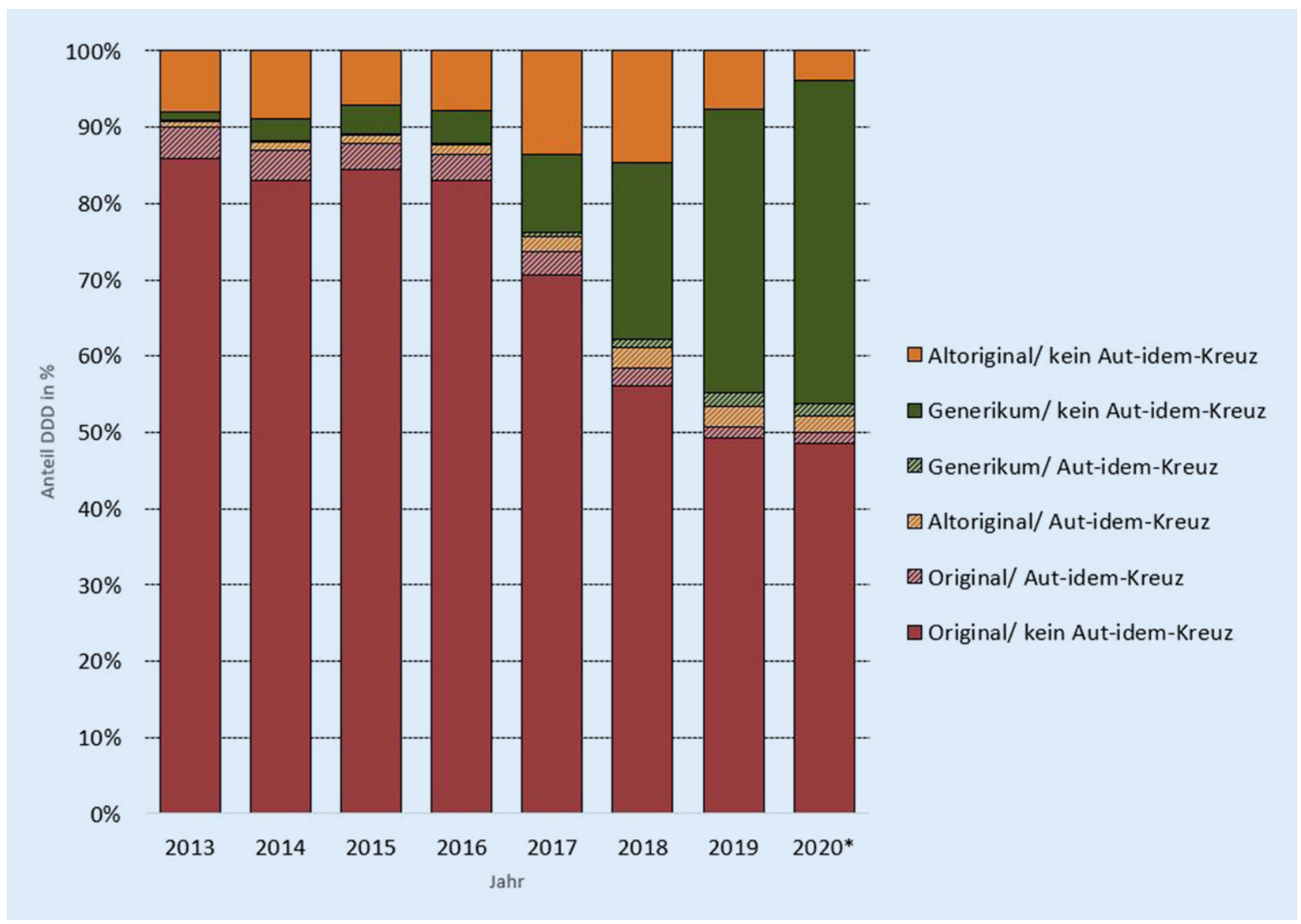

Abb. $2 \Delta$ Bundesweite Generikasituation und Verwendung des Aut-idem-Kreuzes über die Jahre 2013-2020. Summe Defined Daily Dose (DDD) für HIV, inkl. HIV-Präexpositionsprophylaxe (PrEP) und Hepatitis B (HBV). * Daten bis zum 1. Halbjahr 2020. (Datenquelle: Deutsches Arzneiprüfungsinstitut e.V.)

als auch im negativen Sinne auf die Generikaquote auswirken.

Der Blick auf Apothekendaten zeigt, dass nur rund $5 \%$ der generikafähigen Medikamente in den Jahren 2013-2020 als nicht austauschfähig markiert wurden (• Abb. 2). Die verordnenden Ärzte unterstützen somit das Steuerungselement Rabattvertrag. Hierbei ist noch anzumerken, dass nicht nur Altoriginale per Autidem-Kreuz in den Verordnungen gehalten wurden, sondern im Gegenteil auch eine Fixierung auf bestimmte Generika durch Setzen des Aut-idem-Kreuzes stattfand.

Eine andere Möglichkeit der aktiven Einflussnahme ist die Wahl der Wirkstoffe bzw. Medikamente in der Therapie. Betrachten wir daher, in welchem Verhältnis Originale bzw. Altoriginale und Generika in der HIV-Therapie stehen unter Berücksichtigung der in der aktu- ellen Deutsch-Österreichischen Leitlinie zur antiretroviralen Therapie der HIVInfektion empfohlenen Substanzkombinationen [8]. Als generikafähige Stoffe bieten sich hier nach Klassenreihenfolge die NRTI: 3TC, TDF, ABC und die PI: DRV, Atazanavir (ATV) und Ritonavir ( $r$ als Boostersubstanz sowie für den Doppel-NRTI-Backbone (Backbones bilden die Basis einer Kombination, die mit weiteren Substanzen anderer Klassen gemeinsam verabreicht werden): TDF/FTC, ABC/3TC als Kombinationspräparate sowie einzeln kombiniertes 3TC/TDF an. Daraus ergeben sich folgende maximale Generika-zu-OriginalVerhältnisse für die Mehrtabletten-Regime-Kombinationen: NNRTI/NRTI (1:1), INSTI/NRTI (1:1) und PI/NRTI (3:0), da die in den Leitlinien als zu bevorzugende Therapie empfohlenen
NNRTI und INSTI noch nicht generisch verfügbar sind (• Tab. 2).

Würden für den Doppel-NRTI-Backbone statt des generischen Kombinationsmedikaments die Einzelmedikamente genommen, kann pro Regime zulasten der Tablettenanzahl das Verhältnis um eine Generika-DDD gesteigert werden. Patentgeschützte Originale der Gruppe der Kombinationspräparate sind bis auf eine Ausnahme sämtlich Single-TabletRegimen (STR) und stellen jeweils eine nicht generische DDD pro Regime dar. Damit leisten STR einen negativen Beitrag im Sinne der Quote. Besonders ungünstig im Sinne der Generikaquote sind zusammengesetzte Regime mit dem Doppel-NRTI-Backbone, der TenofovirAlafenamid (TAF) enthält. Diese schlagen mit 2 nichtgenerischen DDD negativ zu Buche. 
Tab. 2 HIV-Initialtherapie nach Deutsch-Österreichischen Leitlinien Stand September 2020 [8] und tatsächliches Verhältnis von Original zu Generikum (Ist) sowie durch aufspalten der Kombinationspräparate mögliches Verhältnis (Optional)

\begin{tabular}{|c|c|c|c|c|c|}
\hline \multicolumn{2}{|c|}{ Für die Primärtherapie empfohlen: } & \multirow{2}{*}{$\begin{array}{l}\text { DDD Ist } \\
\text { Original }\end{array}$} & \multirow[b]{2}{*}{ Generisch } & \multicolumn{2}{|c|}{$\begin{array}{l}\text { DDD Optional durch aufspal- } \\
\text { ten }\end{array}$} \\
\hline & & & & Original & Generisch \\
\hline \multicolumn{6}{|c|}{ Eintablettenregime } \\
\hline \multirow{4}{*}{$\begin{array}{l}\text { INSTI- } \\
\text { basiert }\end{array}$} & $\mathrm{BIC/TAF/FTC}$ & 1 & - & - & - \\
\hline & DTG/ABC/3TC & 1 & - & 1 & 1 \\
\hline & DTG/3TC & 1 & - & 1 & 1 \\
\hline & $\mathrm{EVG} / \mathrm{C} / \mathrm{TAF} / \mathrm{FTC}$ & 1 & - & - & - \\
\hline \multirow{3}{*}{$\begin{array}{l}\text { NNRTI- } \\
\text { basiert }\end{array}$} & DOR/TDF/3TC & 1 & - & 1 & 2 \\
\hline & RPV/TAF/FTC & 1 & - & - & - \\
\hline & RPV/TDF/FTC & 1 & - & 1 & 1 \\
\hline Pl-basiert & $\mathrm{DRV} / \mathrm{c} / \mathrm{TAF} / \mathrm{FTC}$ & 1 & - & - & - \\
\hline \multicolumn{6}{|c|}{ Mehrtablettenregime } \\
\hline \multirow{4}{*}{$\begin{array}{l}\text { INSTI- } \\
\text { basiert }\end{array}$} & DTG + TAF/FTC & 2 & - & - & - \\
\hline & DTG + TDF/FTC & 1 & 1 & - & - \\
\hline & $\mathrm{RAL}+\mathrm{TAF} / \mathrm{FTC}$ & 2 & - & - & - \\
\hline & $\begin{array}{l}\mathrm{RAL}+(\mathrm{ABC} / 3 \mathrm{TC} \text { oder } \\
\mathrm{TDF} / \mathrm{FTC})\end{array}$ & 1 & 1 & - & - \\
\hline \multirow{2}{*}{$\begin{array}{l}\text { NNRTI- } \\
\text { basiert }\end{array}$} & $\mathrm{DOR}+\mathrm{TAF} / \mathrm{FTC}$ & 2 & - & - & - \\
\hline & $\begin{array}{l}\text { DOR+ (TDF/FTC oder } \\
\text { ABC/3TC) }\end{array}$ & 1 & 1 & - & - \\
\hline \multirow[t]{2}{*}{ PI-basiert } & $\mathrm{DRV} / \mathrm{r}+\mathrm{TAF} / \mathrm{FTC}$ & 1 & 2 & - & - \\
\hline & $\mathrm{DRV} / \mathrm{r}+\mathrm{ABC} / 3 \mathrm{TC}$ & - & 3 & - & - \\
\hline \multicolumn{6}{|c|}{$\begin{array}{l}\text { Durch Schrägstrich getrennte Substanzen sind in einem Präparat, durch Pluszeichen getrennte in ver- } \\
\text { schiedenen Präparaten enthalten } \\
\text { ABC Abacavir, BIC Bictegravir, c Cobicistat, DRV Darunavir, DDD Defined Daily Dose, DTG Dolutegravir, } \\
\text { DOR Doravirin, EVG Elvitegravir, FTC Emtricitabin, INSTI Integrase-Strang-Transfer-Inhibitoren, } 3 T C \text { Lami- } \\
\text { vudin, NNRTI Non-Nukleos(t)ide-Reverse-Transkriptase-Inhibitoren, PI Proteaseinhibitoren, RAL Ralte- } \\
\text { gravir, r Ritonavir, TAF Tenofovir-Alafenamid, TDF Tenofovir-Disoproxil }\end{array}$} \\
\hline
\end{tabular}

Es kann festgehalten werden, dass zusammengesetzte NNRTI- und INSTIRegime mit Generikabackbone bezüglich der Generikaquote neutral sind, zusammengesetzte PI-Regime die Generikaquote durch Verdopplung oder Verdreifachung der DDD erhöhen bzw. dass diese ggf. 3 STR kompensieren. Daraus folgt, dass die in der KV Bayern (2020) festgesetzte Generikaquote von - für die meisten Arztgruppen grob zusammengefasst - etwa $43 \%$ innerhalb der HIV-Therapieverordnungen nur mit einer ausreichend hohen Quote an zusammengesetzten PI-Regimen erreicht werden kann. Ausgleichend wirkt sich auch eine ausreichende Anzahl an HBVMonoinfizierten und PrEP-Gebrauchern aus, die generisch behandelt werden.

Im Datenkörper des DAPI sind Angaben zur Bindung der verordneten Medikamente an Rabattverträge verfügbar.
Die Abb. 3 und 4 zeigen den bundesweiten Marktstatus und das Vorhandensein von Rabattverträgen.

Mit dem Eintritt generischer Medikamente in den Markt, waren sowohl Verordnungen von rabattierten Generika als auch rabattierten Altoriginalen zu verzeichnen. Der Anteil an Verordnungen mit rabattierten Altoriginalen stieg über die Zeit an. Im Jahr 2016 überstieg das Segment der rabattierten Altoriginale sogar den gesamten Generikaanteil. Mit dem weiteren Anstieg der Generika im Verhältnis zu den Altoriginalen sank der Anteil von Altoriginalen mit Rabattbindung zuletzt in den Jahren 2019 und 2020 stark. Der Anteil von Altoriginalen ohne Rabattvertrag lag 2019 bei $7 \%$ und 2020 bei $5 \%$. Die verordneten Generika waren über die gesamte Zeit fast vollständig Gegenstand von Rabattverträgen. In dem Kontext lassen sich auch
Rückschlüsse auf Markteintrittsstrategien pharmazeutischer Unternehmen ziehen: Bei STR und patentgeschützten $\mathrm{Me}$ dikamenten sind kaum Rabatte erkennbar. Anschließend, also ab generischer Verfügbarkeit, werden in größerem Umfang Rabatte auf das Altoriginal wie auch auf das Generikum gegeben. Im Kontext der jüngsten Einführung einer Festbetragsgruppe (ab Oktober 2020) kam es ebenfalls zum Abschluss von Rabattverträgen.

\section{Diskussion}

Die verordnungssteuernden Maßnahmen in der GKV-Arzneimittelversorgung zeigen auch in der HIV-Schwerpunktversorgung Wirkung. Die in der Vergangenheit gängigen Steuerungsinstrumente, also statistische Auffälligkeitsprüfungen (zuvorderst Richtgrößen mit HIV als Praxisbesonderheit), werden dabei ergänzt oder gar ersetzt durch Quotenlösungen. Eine solche Steuerung setzt nicht beim Faktor Menge, sondern beim Faktor Struktur an. Die konkrete Ausgestaltung kann sehr unterschiedlich sein, dies zeigen die „Pionier“Generikaquoten der KV Bayern und KV Berlin.

Eine Analyse auf Grundlage von GKV-Apothekenabrechnungsdaten verdeutlicht Potenziale, aber auch die Grenzen eines solchen Instrumentes zur Verordnungssteuerung. Vor allem 4 Sachverhalte zeigen sich:

1. Das Quotenpotenzial für Generika im patentfreien Bereich in der Versorgung von Menschen mit HIV wird bereits weitgehend ausgeschöpft. Dieser Umstand ist vor allem darauf zurückzuführen, dass die HIVVerordner den Austausch mittels Verzicht auf Aut-idem-Kreuze unterstützen. Dass der Markteintritt von generischen HIV-Therapeutika im Verordnungsverhalten berücksichtigt wird, zeigen auch Umfragen [1], was im Ergebnis dazu führt, dass Rabattvertragsstrategien von Krankenkassen und Herstellern wirken können. Dadurch wird sowohl einerseits das Altoriginal zeitweise im Markt gehalten als anderseits die Abgabe des Generikums gesichert. 


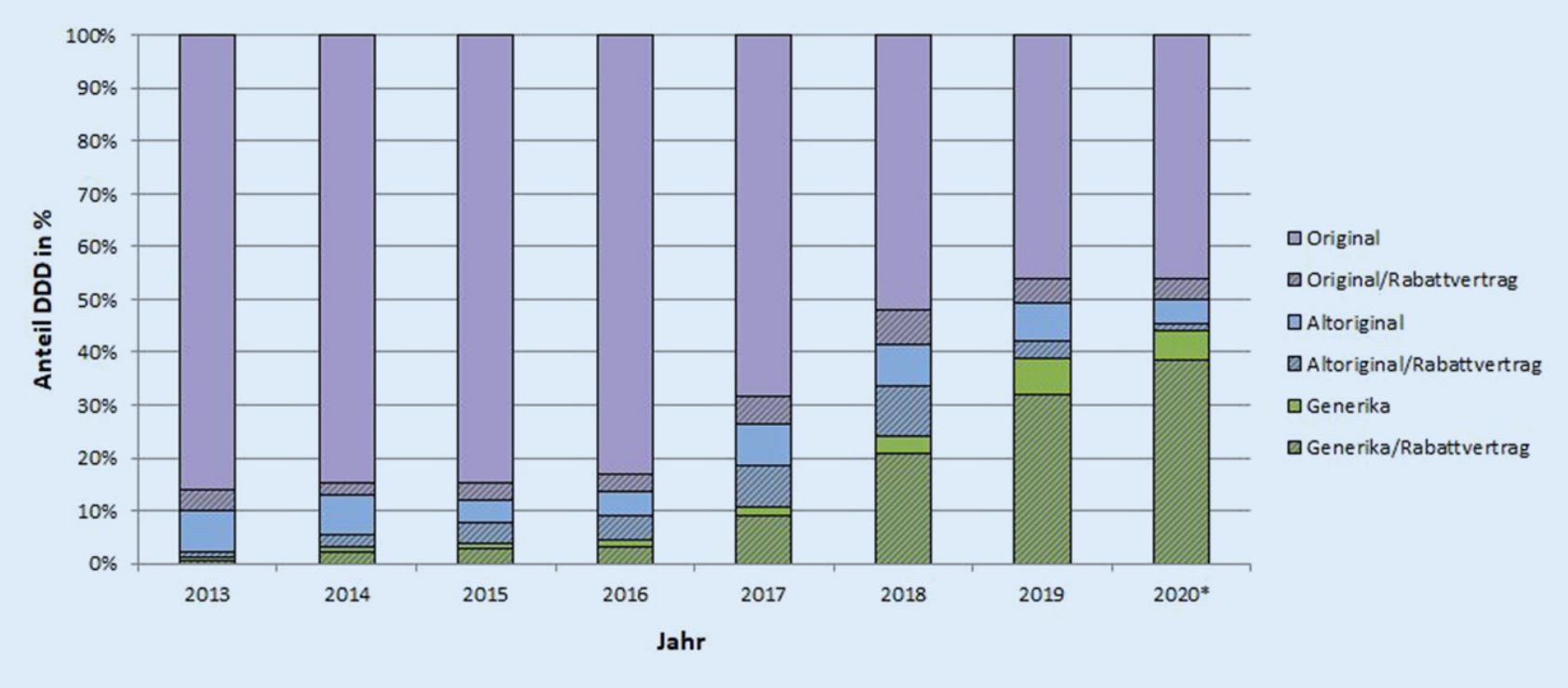

Abb. 3 ॥ Bundesweite Verteilung der Abgaben von Original, Altoriginal und Generika nach Vorliegen von Rabattverträgen über die Jahre 2013-2020. Summe Defined Daily Dose (DDD) für HIV, inkl. HIV-Präexpositionsprophylaxe (PrEP) und Hepatitis B (HBV). * Daten bis zum 1. Halbjahr 2020. (Datenquelle: Deutsches Arzneiprüfungsinstitut e. V.)

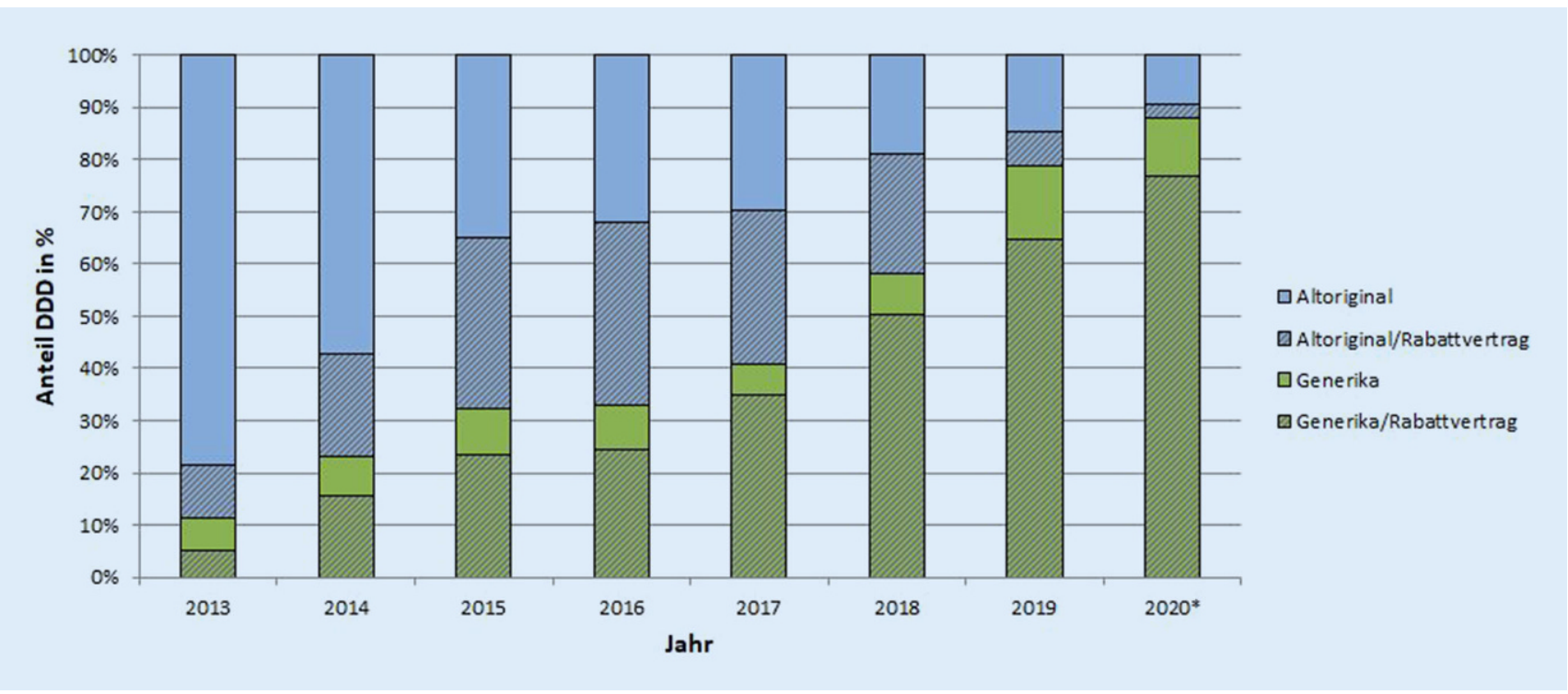

Abb. 4 \ Bundesweite Abgabe bezogen auf den generikafähigen Markt nach Vorliegen von Rabattverträgen über die Jahre 2013-2020. Summe Defined Daily Dose (DDD) für HIV, inkl. HIV-Präexpositionsprophylaxe (PrEP) und Hepatitis B (HBV). * Daten bis zum 1. Halbjahr 2020. (Datenquelle: Deutsches Arzneiprüfungsinstitut e.V.)

Die Nichtverwendung von Aut-idemKreuzen durch die Verordner wirkt hier unterstützend. Grundsätzlich ist es daher sinnvoll, bei der Berechnung der Quoten Rabattverträge einzubeziehen.

2. Solange eine Generikaquote grundsätzlich anstrebt das generikafähige Potenzial auszureizen, d.h. den Anteil der nicht rabattierten Altoriginale zu substituieren, könnte eine (hypothe- tische) bundesweite Generikaquote bezogen auf den Gesamtmarkt HIV, PrEP und HBV um knapp 5\% (von aktuell ca. 44\%) gesteigert werden. Ob ein solcher Schritt mit Blick auf den geringen Preisunterschied zu den nichtrabattierten Altoriginalen wirtschaftlich sinnvoll ist, sei dahingestellt. Die bayerische Wirkstoffvereinbarung mit zumeist $49 \%$
Quotenvorgabe dürfte damit bereits an Grenzen stoßen.

3. Ist man hingegen bereit, auch an die Wahl der verordneten Medikamente selbst heranzugehen, ergeben sich folgende Optionen: Die Generikaquote könnte gesteigert werden durch die "Aufspaltung" von STR in einen generischen Backbone und einen originalen aktiven Teil, ferner durch die Auswahl nicht empfohle- 
ner generischer Klassenvertreter oder durch die Wahl einer Klasse mit hohem generischen DDD-Anteil (PI). Eine Abspaltung des generischen Backbones treibt im Ergebnis die Quote der generischen DDD, ist aber insbesondere nach Etablierung der entsprechenden Festbetragsgruppe wirtschaftlich nicht sinnvoll. Auf den Einspareffekt bezogen gibt es bei einigen Kombinationen keine nennenswerte Kostenersparnis bzw. ist die neue Kombination sogar teurer. Zudem sind die STR, die seit 2011 in den Markt kamen, alle AMNOGbewertet.

4. Die HIV-Generikaquote ist de facto eine erweiterte Quote für den $\mathrm{Ge}$ samtmarkt HIV und HBV - inkl. der PrEP -, d.h., die Berechnung der Generikaquoten spiegelt den entsprechenden Gesamtmarkt wider und sollte inhaltlich und begrifflich entsprechend berücksichtigt werden. Beispielsweise ist die höhere Quote im HIV-Segment in Berlin vermutlich auf den höheren PrEP-Umsatz zurückzuführen.

Durch eine Orientierung an den erreichten Quoten werden diese auf dem Istniveau fixiert und es geht immer nur noch höher herauf (Soll-Zustand). Geht man davon aus, dass die bisherigen Quoten in einer ersten Phase eher dem "Gewöhnen" der Verordner an das neue Steuerungsinstrumentarium dienen und zunächst weitgehend den Ist-Zustand abbilden, stellt sich die Frage, wie ein mögliches Soll aussehen könnte. Wie gezeigt werden konnte, besteht im Bereich der (nichtrabattierten) Altoriginale noch „Luft“. Hier ist allerdings zu bedenken, dass genau dieses Segment mittlerweile in Festbetragsgruppen eingeordnet wurde.

\section{Fazit}

Die Steuerungsinstrumente der Arzneimittelversorgung in der GKV setzen auf indikationsbezogene Quoten, vorrangig Leitsubstanzen und/oder Generika/ Biosimilars. Diese gibt es mittlerweile auch im HIV-Bereich, etwa die seit 2020 in Bayern und Berlin eingeführten
Generikaquoten für HIV-Therapeutika. Ziel des vorliegenden Beitrages ist es, auf Grundlage von GKV-Apothekenabrechnungsdaten das Potenzial sowie Grenzen von Generikaquotenlösungen in der HIV-Versorgung $\mathrm{zu}$ analysieren und Handlungsempfehlungen $\mathrm{zu}$ skizzieren.

Es zeigt sich, dass das Quotenpotenzial für Generika im patentfreien Bereich in der HIV-Versorgung bereits weitgehend ausgeschöpft wird. Dieser Umstand ist vor allem darauf zurückzuführen, dass die HIV-Verordner den Austausch durch Verzicht auf Aut-idem-Kreuze auf dem Kassenrezept unterstützen. Das steuerungspolitische Optimum ist also fast erreicht. Dies ist ein Indiz dafür, dass ein Instrumentarium bestehend aus Rahmenvertrag zur Arzneimittelversorgung sowie einer leitliniengerechten Wirkstoffverordnung durch den Arzt - in Verbindung mit dem AMNOG und den Festbeträgen - steuerungspolitisch gut geeignet ist. Die Leitlinienkonformität und das (existierende) Eintablettenregime müssen aber beibehalten werden, damit die gute Versorgungsqualität gewährleistet bleibt. Die aktuellen DeutschÖsterreichischen Leitlinien streben als oberste Prinzipien virologische und immunologische Wirksamkeit sowie die Vermeidung von Resistenzentwicklung an [8]. Diese Ziele werden durch die Auswahl der ART anhand der Kriterien Anwendungsfreundlichkeit, geringe Einnahmefrequenz, niedrige Tablettenzahl, wenig pharmakokinetische Interaktionen und diätetische Restriktionen positiv beeinflusst.

Ein Austausch zum Erreichen einer höheren Generikaquote führt in jedem Fall zu einer Erhöhung der Tablettenanzahl und widerspricht damit dem Grundsatz der Anwendungsfreundlichkeit, ggf. führt er sogar zur Wahl einer suboptimalen Therapie. Dergestalt wäre eine Steuerung über Generikaquoten ein zu teuer erkaufter Erfolg. Im Tausch für mögliche wirtschaftliche Vorteile drohen dann Qualitätseinbußen in der Versorgung. Des Weiteren muss Raum für die Verordnung innovativer patentgeschützter Präparate bestehen, die das AMNOGVerfahren durchlaufen haben (und damit aus Verordnersicht bei entsprechender
Bewertung als wirtschaftlich gelten sollten [18]), was auch auf eine Absenkung von Quoten hinauslaufen kann. Hierbei sollte eine Quotenregelung therapeutischen Neuerungen und dynamischen Entwicklungen am Markt, nicht zuletzt bei HBV und PrEP (!) sowie einer individuellen Therapiewahl nicht im Weg stehen.

\section{Korrespondenzadresse}

\section{Daniel Schmidt}

Abteilung für Infektionsepidemiologie, Robert Koch-Institut

Seestraße 10, 13353 Berlin, Deutschland

schmidtd@rki.de

Funding. Open Access funding enabled and organized by Projekt DEAL.

\section{Einhaltung ethischer Richtlinien}

Interessenkonflikt. R. Rüsenberg, G. Gradl, M. Schulz, B. Bartmeyer und D. Schmidt geben an, dass kein Interessenkonflikt besteht. A. Baumgarten gibt Beraterund Vortragstätigkeiten für Gilead, Janssen, MSD, ViiV an. S. Mauss gibt Beratertätigkeiten für Gilead, Janssen, MSD, ViiV an. Es wurden zusätzlich zur Beratertätigkeit keine Vortragshonorare oder Reisekosten zu Kongressen gezahlt. C. Kollan ist Kleinanleger bei Herstellern antiretroviraler Medikamente.

Open Access. Dieser Artikel wird unter der Creative Commons Namensnennung 4.0 International Lizenz veröffentlicht, welche die Nutzung, Vervielfältigung, Bearbeitung, Verbreitung und Wiedergabe in jeglichem Medium und Format erlaubt, sofern Sie den/die ursprünglichen Autor(en) und die Quelle ordnungsgemäß nennen, einen Link zur Creative Commons Lizenz beifügen und angeben, ob Änderungen vorgenommen wurden.

Die in diesem Artikel enthaltenen Bilder und sonstiges Drittmaterial unterliegen ebenfalls der genannten Creative Commons Lizenz, sofern sich aus der Abbildungslegende nichts anderes ergibt. Sofern das betreffende Material nicht unter der genannten Creative Commons Lizenz steht und die betreffende Handlung nicht nach gesetzlichen Vorschriften erlaubt ist, ist für die oben aufgeführten Weiterverwendungen des Materials die Einwilligung des jeweiligen Rechteinhabers einzuholen.

Weitere Details zur Lizenz entnehmen Sie bitte der Lizenzinformation auf http://creativecommons.org/ licenses/by/4.0/deed.de.

\section{Literatur}

1. dagnä (2020) Mitgliederbefragung 2020. Unveröffentlichtes Dokument

2. Stoll M (2020) Tenofovir-Alafenamid: Noch nicht generisch aber schon Festbetrag? In: HIV\&more 3/2020. https://www.hivandmore.de/archiv/ 
2020-3/HIV-m3 20 Akt_Stoll_Festbetrag_.pdf. Zugegriffen: 10. März 2021

3. Heiden $M$ an der, Marcus U, Kollan C, Schmidt D, Gunsenheimer-Bartmeyer B, Bremer V (2020) Schätzung der Zahl der HIV-Neuinfektionen und der Gesamtzahl von Menschen mit HIV in Deutschland, Stand Ende 2019. Epidemiol Bull 48:3-15. https://doi.org/10.25646/7213. Zugegriffen: 10. März 2021

4. BMG (2020a) Gesetzliche Krankenversicherung: Kennzahlen und Faustformeln. https://www. bundesgesundheitsministerium.de/fileadmin/ Dateien/3_Downloads/G/GKV/KF2020Bund_ Maerz 2020.pdf.Zugegriffen: 10. März 2021

5. Valbert F, Wolf E, Schewe K, Klauke S, Hanhoff N, Hoffmann C, Preis S, Pahmeier K, Wasem J, Neumann A (2020) Cost of HIV and determinants of health care costs in HIV-infected treatmentnaive patients initiated on antiretroviral therapy in Germany: experiences of the PROPHET study. Value Health 23(10):1324-1331

6. Hoffmann C(2020) ART 2020/2021.In:HoffmannC, Rockstroh J (Hrsg) HIV 2020/2021. Medizin Fokus Verlag, Hamburg, S42-246

7. Zöllner E, Pieloth K (2019) HIV: Von einer tödlichen zur chronischen Erkrankung. Monit Versorgungsforsch 12(2):14-15

8. DAIG (2020) Deutsch-Österreichische Leitlinien zur antiretroviralen Therapie der HIV-Infektion. https://daignet.de/site-content/hiv-leitlinien/ leitlinien-1/deutsch-oesterreichische-leitlinienzur-antiretroviralen-therapie-der-hiv-infektion. Zugegriffen: 10. März 2021

9. Stoll M (2018) So senken Generika die Kosten der ART. MMW Fortschr Med 160(S2):37-39

10. Lottes M, Schmidt D, Kollan C (2019) Wie viel Generikaquote verträgt die HIV-Behandlung? In: HIV\&more 2/2019. https://www.hivandmore.de/ archiv/2019-2/HIV-m2_19_Akt_RKI_Gen_Quote. pdf.Zugegriffen: 10. März 2021

11. BMG (2020b) Gesetzliche Krankenversicherung: Vorläufige Rechnungsergebnisse 1. bis 4. Quartal 2019. Stand: 11. März 2020. https://www.bundesgesundheitsministerium. de/fileadmin/Dateien/3_Downloads/Statistiken/ GKV/Finanzergebnisse/KV45_1-4_Quartal_ 2019_Internet.pdf.Zugegriffen: 10. März 2021

12. Bauckmann J, Laitenberger U, Schröder M, Telschow C (2017) Rabattverträge. In: Schwabe U, Paffrath D, Ludwig W-D, Klauber J (Hrsg) Arzneiverordnungsreport 2017. Aktuelle Daten, Kosten, Trends und Kommentare. Springer, Berlin, S 181-194

13. Steimle T, Schoch, Goentje-Gesine (2020) Krankenkassen-Management. In: Tunder R (Hrsg) Market Access Management für Pharma- und Medizinprodukte. Instrumente, Verfahren und Erfolgsfaktoren. Springer Gabler, Wiesbaden, $\mathrm{S}$ 415-431

14. Rheinisches Ärzteblatt (2016) Vereinbarung über das Arznei- und Verbandmittelausgabenvolumen für das Kalenderjahr 2016. https://www.aekno.de/ fileadmin/user_upload/RheinischesAerzteblatt/ Ausgaben/2016/2016.01.095.pdf. Zugegriffen: 10. März 2021

15. KV Bayerns (2020) Wirkstoffvereinbarung vom 01.01.2020. https://www.kvb.de/fileadmin/kvb/ dokumente/Praxis/Rechtsquellen/S-Z/KVB-RQWirkstoffvereinbarung-2020.pdf. Zugegriffen: 10 März 2021

16. KV Berlin (2020) Anlage 2 zur Arzneimittelvereinbarung für das Jahr 2020 zwischen der KV Berlin und den Verbänden der Krankenkassen. https://www.kvberlin.de/fileadmin/ user_upload/vertraege kv berlin/arznei und_heilmittelvereinbarungen/arzneimittel vereinbarung2020anl2.pdf. Zugegriffen: 10. März 2021

17. dagnä / AOK Rheinland/Hamburg (2020) Mehr Beratung und Pflegeangebote: Ältere Menschen mit HIV-Infektionen in Nordrhein erhalten künftig mehr Leistungen. Presseinformation vom 29. September.https://www.dagnae.de/wp-content/ uploads/2020/09/59_PM_HIV_Versorgung.pdf. Zugegriffen: 10. März2021

18. Baumgarten A (2014) Erstmals qualitätsgestützte HCV-Behandlung aus Spezialisten-Hand. Interview mit Dr. med. Axel Baumgarten. Connexi 2(6):9-11 